
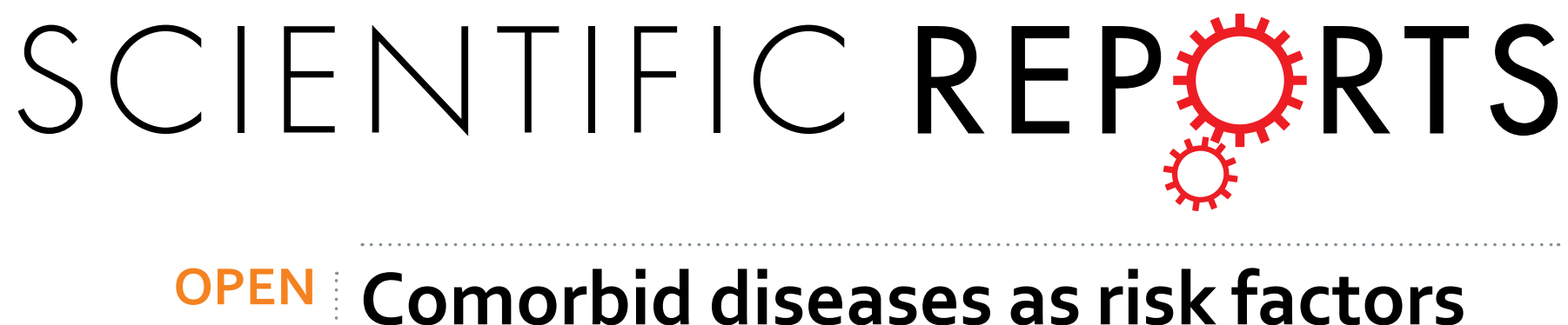

\title{
Comorbid diseases as risk factors for incident posttraumatic stress disorder (PTSD) in a large
}

Received: 15 July 2016

Accepted: 16 December 2016

Published: 27 January 2017

\section{community cohort (KCIS no.PSY4)}

Jung-Chen Chang ${ }^{1}$, Amy Ming-Fang Yen ${ }^{2}$, Hsiu-Hsi Chen ${ }^{3}$, Sam Li-Sheng Chen ${ }^{2}$, SherryYueh-Hsia Chiu ${ }^{4}$, Jean Ching-Yuan Fann ${ }^{5}$ \& Chau-Shoun Lee ${ }^{6,7}$

Nature disasters and terrorist attacks have occurred globally in recent years. Posttraumatic stress disorder (PTSD) has gained increasing attention, but its incidence and comorbidities in the general population are different from those inside the disaster areas. The present study estimated incident PTSD and comorbid diseases for over a decade in a cohort from a community-based integrated screening program. Factors associated with the incidence of PTSD were analyzed using Cox regression models. PTSD incidence was estimated as 81 per $10^{5}$ person-years. Incidence was higher in females than in males and one-year increments in age lowered the risk for PTSD by $3 \%$. Adjusting for other factors, cardiovascular heart disease (adjusted hazard ratio $(\mathrm{aHR})=1.45$, 95\% confidence interval $(\mathrm{Cl}): 1.03-$ 2.04), bipolar disorder ( $\mathrm{aHR}=1.86,95 \% \mathrm{Cl}: 1.07-3.24$ ) and major depressive disorder ( $\mathrm{aHR}=7.03,95 \%$ CI: $5.02-9.85$ ) all significantly increased $45 \%, 86 \%$ and $603 \%$, respectively, the risk of developing PTSD. The low rate of people with incident PTSD receiving treatment in this community health screening population implies there is room for improvement in terms of early detection and intervention. Clinical preventive efforts may be made for patients seeking general medical help, especially those with cardiovascular disorders or mood disorders.

Posttraumatic stress disorder (PTSD) is a common, debilitating mental disorder that describes a syndrome that can occur following traumatic experiences. Lifetime exposure to trauma may reach $50 \%$ or higher ${ }^{1,2}$. Previously reported 1-year and lifetime prevalence rates of PTSD among adults from general population were around 3.5\% and $7 \%$, respectively ${ }^{3-6}$. However, most previous epidemiological studies have reported the prevalence of PTSD in subjects exposed to war, terrorism, or natural disasters, rather than incidence of the disorder in the general population ${ }^{7}$.

The incidence of PTSD varies across different studies and populations. For example, PTSD incidence rates observed among individuals who survived a cardiac arrest range from $19 \%$ to $27 \%$, with comorbid depression and anxiety commonly seen ${ }^{8}$. Among train drivers who had witnessed a railway suicide (a person under the train) the incidence of PTSD ranged from $14 \%$ (severe PTSD) to 44\% (moderate PTSD) ${ }^{9}$. In survivors of motor vehicle accidents, incidences of PTSD were $7.5 \%$ in face-to-face interviews ${ }^{10}$ and $8.5 \%$ in telephone interviews ${ }^{11}$.

The incidence of PTSD also varies geographically ${ }^{2,12}$. In particular, low prevalence rates $(0.2-0.4 \%)$ have been observed in Japan and China ${ }^{13-16}$. The PTSD incidence rate in a Japanese study was much lower than those reported in the United States, United Kingdom, Israel, and Australia, but similar to that in Switzerland ${ }^{17-23}$. It is possible that discrepancies in the incidence of PTSD between developed and developing countries stem from differences in health care, medical advances, and social norms about caring for people following traumatic events

${ }^{1}$ School of Nursing, College of Medicine, National Taiwan University, Taipei, Taiwan; National Taiwan University Hospital, Taipei, Taiwan. ${ }^{2}$ School of Oral Hygiene, College of Oral Medicine, Taipei Medical University, Taipei, Taiwan. ${ }^{3}$ Division of Biostatistics, Graduate Institute of Epidemiology and Preventive Medicine, College of Public Health, National Taiwan University, Taipei, Taiwan. 'Department of Health Care Management, College of Management, Chang Gung University, Taoyuan, Taiwan. ${ }^{5}$ Department of Health Industry Management, College of Healthcare Management, Kainan University, Taoyuan County, Taiwan. ${ }^{6}$ Department of Medicine, Mackay Medical College, New Taipei City, Taiwan. ${ }^{7}$ Department of Psychiatry, Mackay Memorial Hospital, Taipei, Taiwan. Correspondence and requests for materials should be addressed to C.-S.L. (email: csleepsyc@gmail.com) 
or differences in reporting symptoms or seeking care for PTSD. There have been fewer studies of incidence than prevalence of PTSD, and as such, risk factors associated with incident PTSD have not been well investigated. However, age at traumatic event, female gender, (family) history of psychiatric illness, and lower education level are all reported as pre-traumatic risk factors across trauma types ${ }^{24,25}$.

As it is often not feasible to collect data before traumatic events have occurred, investigations of risk factors for incident PTSD are often retrospective rather than prospective ${ }^{26,27}$. There is also a wide range of common comorbidities for incident PTSD, including mental illnesses, such as depressive disorders, anxiety, and substance-related disorders, and physical diseases, such as diabetes, obesity, angina, hypertension, gastritis, and arthritis ${ }^{6,28-31}$. However, although antecedent physical conditions, such as metabolic syndrome and cardiovascular disorders, have been shown to be associated with various psychiatric disorders ${ }^{32,33}$, these have not been well studied in PTSD.

In this study, we aimed to prospectively investigate mental and physical comorbid diseases associated with incident PTSD in a cohort participating in a community health-screening program. The presence of such potential risk factors is routinely assessed in healthcare settings before the onset of PTSD. If comorbid diseases increase the risk of incident PTSD, enhanced surveillance in high-risk populations may be warranted.

\section{Methods}

Study participants and procedure. The cohort was derived from a population of about 390 thousands adults (aged 30 years and above) in the northeastern part of Taiwan. The population was invited to participate in a longitudinal community-based study, the Keelung Community-based Integrated Screening (KCIS), between 1999 and 2004. The details of KCIS have been described elsewhere ${ }^{34}$. Briefly, the KCIS program was originally designed as a continuing evidence-based screening program for five neoplastic and three non-neoplastic diseases (type 2 diabetes, hypertension, and hyperlipidemia) with inter-screening intervals ranging from 1 to 5 years (mean: 3.13 , $\mathrm{SD}:$ 1.47). As the KCIS program is a multiple screening program, the follow-up of this cohort enabled us to ascertain multiple outcomes on neoplastic diseases and other chronic disorders, such as mental illnesses (including PTSD).

We excluded participants with a current or previous diagnosis of PTSD at baseline. Individuals at-risk were followed up from 1999 to 2004 to calculate the incidence of PTSD. In this prospective design, each participant entered the study at some point between 1999 and 2004, and the follow-up period depended on the entrance date. The coverage rate (percentage of participation) of residents enrolled into the KCIS program was approximately $55 \%$ in $2004^{35}$. As a result, there were approximately 138,420 target population eligible for inclusion, yielding 76,545 at-risk participants for the PTSD incidence survey. Of these, 76,417 completed data collection for the final analysis. The exclusion of 128 subjects was due to missing data on essential variables, such as those about clinical diagnoses, metabolic components, and life style details.

Community nurses administrated questionnaires, which inquired about social demographics and life style details (e.g., smoking or alcohol drinking habits etc.) that might be associated with PTSD. Participants were not aware of any hypothesized associations between the risk factors and PTSD before screening. The study hypothesis was formulated independent from PTSD and comorbid aliments ascertainment.

Data Collection. On entering the program, enrolled participants were interviewed to collect social demographics, health behaviors (e.g., smoking or alcohol drinking), and family history of major illnesses (e.g., psychiatric disorders). Assessment of smoking and alcohol consumption was dichotomous: current use or no current use. Further examinations included reclining blood pressure (BP) after 5 to 10 minutes of rest and a fasting blood sample to assess glucose, triglyceride (TG), and high-density lipoprotein (HDL) cholesterol levels. Trained staff measured participants' heights using a stadiometer, waist circumference (WC, to the nearest $0.1 \mathrm{~cm}$ ) by a standard tape, and body weight (to the nearest $0.1 \mathrm{~kg}$ ) by a standardized weight scale. The waist size was checked horizontally at the midway between the inferior margin of the rib cage and the iliac crest.

Measurement of metabolic syndrome (MetS) and its components. MetS was diagnosed according to the NCEP ATP III criteria ${ }^{36}$ with the adjustment for waist size in Asian subjects ${ }^{37}$. We defined participants as having MetS if three or more of the following criteria were met: (1) central obesity (an increased WC, WC $\geq 80 \mathrm{~cm}$ for women and $\geq 90 \mathrm{~cm}$ for men); (2) high TG (TG $\geq 150 \mathrm{mg} / \mathrm{dL}$ ); (3) a low HDL cholesterol concentration $(<50 \mathrm{mg} / \mathrm{dL}$ for women and $<40 \mathrm{mg} / \mathrm{dL}$ for men); (4) an elevated BP ( $\geq 130 \mathrm{mmHg}$ systolic or $\geq 85 \mathrm{mmHg}$ diastolic); and (5) glucose intolerance (elevated fasting glucose $\geq 110 \mathrm{mg} / \mathrm{dL}$ ).

Diagnosis of PTSD. Data used for identifying patients with PTSD were drawn from a national population-based dataset, the National Health Insurance Dataset (NHID). In 1995, a government supported single-payer national health care system was implemented in Taiwan. This system offers affordable and rapid medical attention. National Health Insurance, one of the most complete health insurance systems in the world, finances healthcare for almost all (>99\%) 23 million Taiwanese citizens. The NHID provides details on medical visits, including International Classification of Diseases (ICD) codes, dates of visits, and so on. This universal NHID offers a comparable prognosis with an affordable premium relative to other large series of health insurance datasets in developed countries. In the present study, individuals in the NHID were diagnosed with PTSD according to the ICD-9 (codes 309s), ascertained by psychiatrists' or physicians' clinical diagnosis.

Information on comorbid medical and mental disorders. To assess diseases that are comorbid with PTSD, we retrieved data on several major illnesses from the NHID, which diagnoses were ascertained by psychiatrist or physician in each specialty. This included neoplasm (ICD codes 140-208), diabetes mellitus (DM, 250), hypertensive disorders (HTN, 401-405), cardiovascular diseases (CVD, 390-398, 410-414, and 420-429), and cerebrovascular accidents (CVA, 430-438). Mood-related psychiatric disorders, relevant to the development of 


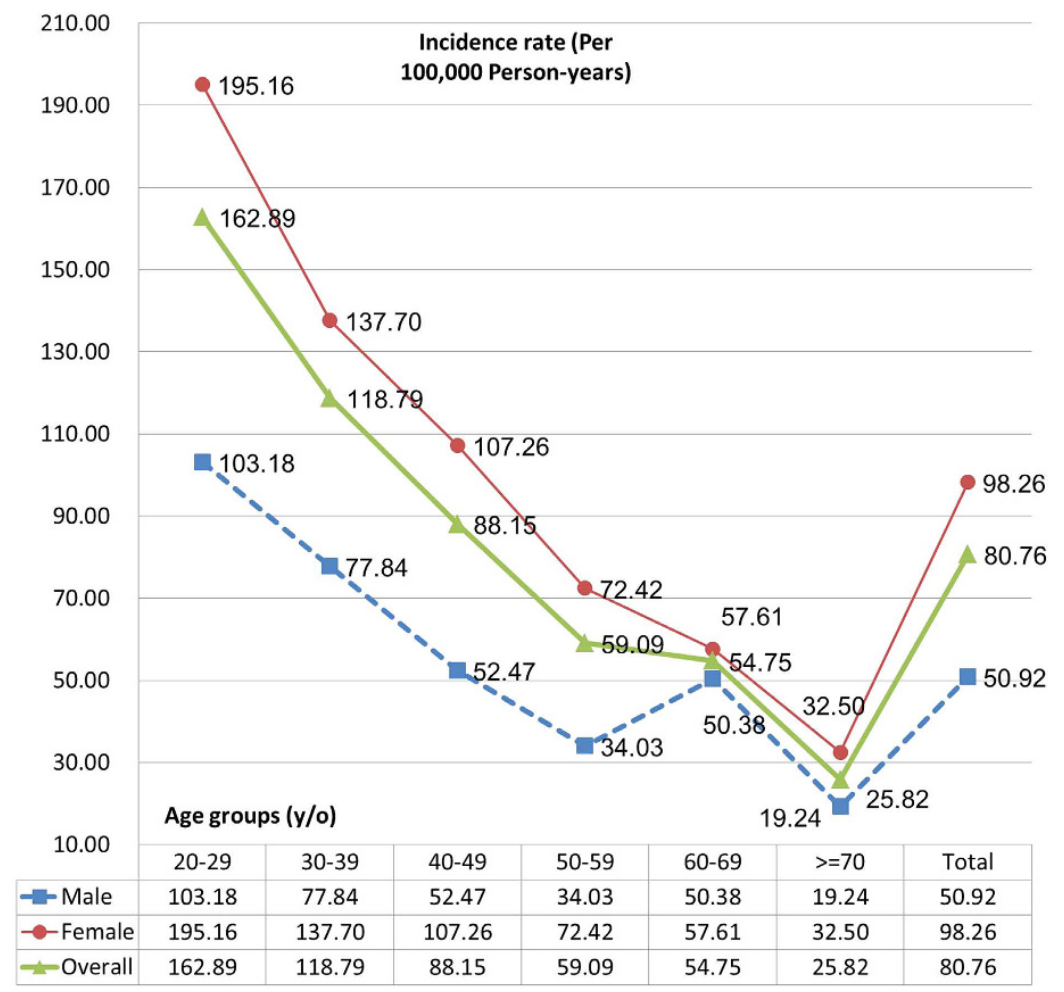

Figure 1. Age- and gender-specific incidence of PTSD.

PTSD, were enrolled for this investigation. We defined major depressive disorders (MDD) according to the ICD diagnostic codes 296.20-296.26, 296.30-296.36, and 296.82. Bipolar disorders (BPD) were defined according to the codes 296.00 to $296.06,296.10$ to $296.16,296.40$ to $296.46,296.50$ to $296.56,296.60$ to $296.66,296.7,296.80$, $296.81,296.89$, and 296.90). Anxiety disorders were defined according to the codes 300.0x. Substance use disorders (SUD) were defined according to the codes 291 s, 292 s, 303 s, 304 s, and 305 s. The comorbid medical and mental disorders were clinically diagnosed by a psychiatrist or physician based on ICD or DSM (Diagnostic and Statistical Manual of Mental disorders) criteria. Information on medical history was obtained from the NHID. To ensure the validity of diagnoses, diseases were identified according to the respective ICD-9 codes at least three times outpatient visits or one hospital admission.

Statistical analyses. For descriptive analyses, continuous variables were expressed as means $( \pm S D)$ and categorical variables were expressed as percentages. To calculate the incidence of PTSD, person-years at risk since the date of attending the first screening were calculated. Eligible participants ceased to contribute to person-years at census either after their final recorded assessment or when they met the criteria for PTSD.

We first used univariate Cox proportional hazards regression models to estimate the hazard ratio of developing PTSD with demographic and clinical characteristics as covariates. A multivariable Cox regression model was further conducted with a stepwise selection method set at 0.10 for inclusion or exclusion of variables within each criterion. Finally, two-tailed tests with $\alpha=0.05$ were considered statistically significant. All statistical analyses were carried out using SAS software (version 9.2; SAS Institute Inc, Cary, NC).

Ethical consideration and funding source. We used data derived from a large research project (KCIS) $)^{34}$. The original research protocol was reviewed and approved by the ethical review committee of National Taiwan University. The KCIS program performs annual recruitment screenings, which are approved by the local ethical committee in the Health Bureau of Keelung City. These approvals include data linkage systems and strict maintenance of participant confidentiality. All methods were performed in accordance with the relevant guidelines and regulations.

Written informed consent was obtained from each participant at the time of recruitment to the program ${ }^{34}$. Because the personal identification numbers for the datasets were encoded, the privacy and confidentiality of patients were ensured by obscuring the links between datasets. None of the authors received outside funding for the current study.

Results

Cumulative incidence of PTSD. Among 76,417 at-risk subjects followed until the end of 2004, 193 incident PTSD cases were found. Of these, 148 were female (76.7\%). The average age of PTSD cases was 43.5 (S.D. 13.8). The overall incident rate of PTSD was $0.08 \%(95 \% \mathrm{CI}=0.06-0.10 \%)$. Figure 1 shows age- and gender-specific incidences of PTSD. Incidence of PTSD was higher for female than male participants across all age groups. Subjects aged 20 to 29 years had the highest incidence of PTSD for both genders (detailed statistics are shown in Table 1). 


\begin{tabular}{|l|c|c|c|c|c|c|c|c|c|}
\hline \multirow{2}{*}{$\begin{array}{l}\text { Age } \\
\text { (years) }\end{array}$} & \multicolumn{4}{|c|}{ Male } & \multicolumn{4}{c|}{ Female } & \multicolumn{3}{|c|}{ Both genders } \\
\cline { 2 - 11 } & Cases & PY & $\begin{array}{c}\text { Incidence } \\
\left(\times \mathbf{1 0}^{-5}\right)\end{array}$ & Cases & PY & $\begin{array}{c}\text { Incidence } \\
\left(\times \mathbf{1 0}^{-5}\right)\end{array}$ & Cases & PY & $\begin{array}{c}\text { Incidence } \\
\left(\times \mathbf{1 0}^{-5}\right)\end{array}$ \\
\hline $20-29$ & 6 & 5815 & 103.18 & 21 & 10760 & 195.16 & 27 & 16576 & 162.89 \\
\hline $30-39$ & 12 & 15417 & 77.84 & 46 & 33407 & 137.70 & 58 & 48824 & 118.79 \\
\hline $40-49$ & 11 & 20966 & 52.47 & 42 & 39158 & 107.26 & 53 & 60124 & 88.15 \\
\hline $50-59$ & 5 & 14692 & 34.03 & 20 & 27615 & 72.42 & 25 & 42307 & 59.09 \\
\hline $60-69$ & 8 & 15880 & 50.38 & 14 & 24299 & 57.61 & 22 & 40179 & 54.75 \\
\hline$>=70$ & 3 & 15596 & 19.24 & 5 & 15385 & 32.50 & 8 & 30981 & 25.82 \\
\hline Total & 45 & 88367 & 50.92 & 148 & 150625 & 98.26 & 193 & 238991 & 80.76 \\
\hline
\end{tabular}

Table 1. Age- and gender-specific incidence of PTSD in a large community longitudinal cohort $(\mathbf{n}=76,417)$. PY = person-years.

In the univariate logistic regression, compared with non-PTSD participants, those with PTSD tended to be female, younger, more highly educated, non-smokers, and non-drinkers. Furthermore, participants with PTSD had a higher proportion of CVD, MDD, anxiety disorders, BPD, and SUD. MetS, however, demonstrated a minor protective effect for the occurrence of PTSD (Table 2).

Risk factors for developing PTSD at follow-up. Table 3 shows the results of the univariate Cox proportional hazard regression models, indicating that male gender and older age were significantly inversely associated with PTSD incidence. Therefore, we subsequently controlled for the effects of sex and gender, and found that the onset of PTSD was significantly associated with low education, physical illnesses (cancer, diabetes, hypertension, CVA, and CVD), and mental disorders (MDD, anxiety disorders, BPD, and SUD).

The multivariable Cox regression analysis showed that MDD accounted for around a 7-fold increase in risk for developing PTSD ( $\mathrm{aHR}=7.03,95 \% \mathrm{CI}=5.02-9.85)$. Bipolar disorders were also associated with the incident PTSD $(\mathrm{aHR}=1.86,95 \% \mathrm{CI}=1.07-3.24)$. CVD increased incident PTSD risk by $45 \%(95 \% \mathrm{CI}=1.03-2.04)$. One-year increments in age lowered the risk of PTSD by $3 \%(\mathrm{aHR}=0.97,95 \% \mathrm{CI}=0.95-0.98)$. Education level was significantly associated with PTSD, with those educated to elementary school or lower having half the risk of PTSD compared with those educated to college or above $(\mathrm{aHR}=0.54,95 \% \mathrm{CI}=0.32-0.90)$.

\section{Discussion}

The strengths of this study include the large sample size from a general population, long follow-up period, and linked data from multiple sources. In this large community cohort, we found an overall incident rate of PTSD as low as $0.08 \%$. However, various physical and psychological antecedents were common in the development of PTSD. In our multivariable analyses, MDD accounted for a greater than 6-fold increase in hazard ratio for PTSD and BPD and CVD increased the hazard ratio by around $86 \%$ and $45 \%$, respectively. MetS was not found to be associated with the development of PTSD after adjustment for covariates.

We identified incident cases among those receiving clinical diagnoses and healthcare services. The cumulative incidence rate in our study is relatively low, but is consistent with rates of PTSD after a traumatic experience reported in another Eastern society ${ }^{15,16}$ and in Asian Americans ${ }^{38}$. In a survey of common psychiatric disorders among a nationally representative sample of Taiwanese, similarly low rates of MDD were also reported ${ }^{39}$. One possible explanation for this is the low level of help-seeking behavior for mental disorders commonly seen in Asian countries $^{40}$ and the limited understanding of PTSD in the general public. When PTSD affects US ethnic minorities, the mental illness is usually undertreated, especially in Asian groups ${ }^{38}$. This indicates room for improvement in the detection of PTSD in health screening programs in Asian countries ${ }^{41}$. Alternatively, under-diagnosis may also arise from the insufficient awareness of PTSD in clinicians ${ }^{39,41}$. In a recent World Mental Health (WMH) Surveys in 18 countries, disaster-related PTSD prevalence was only $0.0-3.8 \%$ and was significantly related to high education and other risk factors ${ }^{42}$. The present study also found low rate of PTSD and related to higher educated persons. It may be explained by high level of awareness of PTSD and willingness to seek help from medical professionals in higher than lower educated persons.

Associations between CVD and PTSD have been consistently reported in the general population ${ }^{43}$. Possible mechanisms for this link include cytokines, increased allostatic load, the hypothalamic-pituitary-adrenal axis, the autonomic nervous system, psychiatric risk factors (e.g., substance abuse, depression), shared genetic vulnerability, and MetS ${ }^{44-49}$. However, most studies have investigated the impact of PTSD on the risk or outcome of $\mathrm{CVD}^{50}$. In the present study, we investigated the relationship in the opposite direction to find that CVD played a role in the development of PTSD. Recent evidence has also highlighted the bidirectional relationship between PTSD and $\mathrm{CVD}^{44}$. Life-threatening medical illness can elicit PTSD, and conversely, PTSD increases subsequent CVD events and mortalities. A common physical basis for CVD and PTSD, particularly hyperarousal symptoms, is sympathetic activation, as well as higher levels of coronary artery calcium ${ }^{51}$. Another possible pathway between cardiovascular events and PTSD is medication nonadherence causing exacerbated physiological conditions and an unhealthy lifestyle, such as sleep disturbances ${ }^{52}$.

The co-occurrence of PTSD and depressive disorders is common ${ }^{53,54}$. However, the relational direction and nature of this association is unclear. It has been suggested that these phenomena have overlapping symptoms with similar trait vulnerability. In our longitudinal study, MDD was found to predict the onset of PTSD. Having 


\begin{tabular}{|c|c|c|c|c|c|c|}
\hline Variables & & With PTSD & & Without PTSD & & p-value \\
\hline \multirow{2}{*}{ Gender } & Male & 45 & $0.15 \%$ & 29180 & $99.85 \%$ & \multirow{2}{*}{$<0.0001$} \\
\hline & Female & 148 & $0.31 \%$ & 47044 & $99.69 \%$ & \\
\hline Age (Mean (SD)) & years & $43.5(13.8)$ & & $49.6(15.3)$ & & $<0.0001$ \\
\hline \multirow{3}{*}{ Education } & Low & 45 & $0.15 \%$ & 29169 & $99.85 \%$ & \multirow{3}{*}{$<0.0001$} \\
\hline & Medium & 95 & $0.29 \%$ & 32522 & $99.71 \%$ & \\
\hline & High & 53 & $0.36 \%$ & 14492 & $99.64 \%$ & \\
\hline \multirow{3}{*}{ Marriage } & Single & 26 & $0.24 \%$ & 10682 & $99.76 \%$ & \multirow{3}{*}{0.2545} \\
\hline & Married & 138 & $0.24 \%$ & 56915 & $99.76 \%$ & \\
\hline & Widow & 29 & $0.34 \%$ & 8574 & $99.66 \%$ & \\
\hline \multirow{2}{*}{ Smoking } & No & 157 & $0.29 \%$ & 54075 & $99.71 \%$ & \multirow{2}{*}{0.0006} \\
\hline & Yes & 31 & $0.15 \%$ & 20801 & $99.85 \%$ & \\
\hline \multirow{2}{*}{ Drinking } & No & 163 & $0.28 \%$ & 57105 & $99.72 \%$ & \multirow{2}{*}{0.0007} \\
\hline & Yes & 24 & $0.14 \%$ & 17410 & $99.86 \%$ & \\
\hline \multirow{2}{*}{ Cancer } & No & 173 & $0.25 \%$ & 70330 & $99.75 \%$ & \multirow{2}{*}{0.172} \\
\hline & Yes & 20 & $0.34 \%$ & 5894 & $99.66 \%$ & \\
\hline \multirow{2}{*}{ Diabetes } & No & 149 & $0.25 \%$ & 59532 & $99.75 \%$ & \multirow{2}{*}{0.7629} \\
\hline & Yes & 44 & $0.26 \%$ & 16692 & $99.74 \%$ & \\
\hline \multirow{2}{*}{ Hypertension } & No & 130 & $0.26 \%$ & 49864 & $99.74 \%$ & \multirow{2}{*}{0.5715} \\
\hline & Yes & 63 & $0.24 \%$ & 26360 & $99.76 \%$ & \\
\hline \multirow{2}{*}{ CVA } & No & 170 & $0.25 \%$ & 68310 & $99.75 \%$ & \multirow{2}{*}{0.4852} \\
\hline & Yes & 23 & $0.29 \%$ & 7914 & $99.71 \%$ & \\
\hline \multirow{2}{*}{ CVD } & No & 122 & $0.23 \%$ & 53589 & $99.77 \%$ & \multirow{2}{*}{0.0313} \\
\hline & Yes & 71 & $0.31 \%$ & 22635 & $99.69 \%$ & \\
\hline \multirow{2}{*}{ MetS } & No & 174 & $0.28 \%$ & 62434 & $99.72 \%$ & \multirow{2}{*}{0.0022} \\
\hline & Yes & 18 & $0.13 \%$ & 13543 & $99.87 \%$ & \\
\hline \multirow{2}{*}{ MDD } & No & 130 & $0.18 \%$ & 72117 & $99.82 \%$ & \multirow{2}{*}{$<0.0001$} \\
\hline & Yes & 63 & $1.51 \%$ & 4107 & $98.49 \%$ & \\
\hline \multirow{2}{*}{ ANX } & No & 182 & $0.24 \%$ & 74686 & $99.76 \%$ & \multirow{2}{*}{0.0003} \\
\hline & Yes & 11 & $0.71 \%$ & 1538 & $99.29 \%$ & \\
\hline \multirow{2}{*}{ SUD } & No & 187 & $0.25 \%$ & 75510 & $99.75 \%$ & \multirow{2}{*}{0.0018} \\
\hline & Yes & 6 & $0.83 \%$ & 714 & $99.17 \%$ & \\
\hline \multirow{2}{*}{ BPD } & No & 170 & $0.23 \%$ & 74745 & $99.77 \%$ & $<0000$ \\
\hline & Yes & 16 & $1.07 \%$ & 1479 & $98.93 \%$ & $<0.0001$ \\
\hline
\end{tabular}

Table 2. Demographic and clinical characteristics by the status of PTSD in the univariate logistic

regression. 1 Education: Elementary schools or below (low); High school (medium); University (high). 2 CVA: cerebrovascular accidents; CVD: cardiovascular diseases; MetS: metabolic syndrome; MDD: major depressive disorders; ANX: anxiety disorders; SUD: substances use disorders; BPD: bipolar disorders.

comorbid depression was one of the major risk factors for newly diagnosed PTSD among women who have been sexually assaulted ${ }^{55}$. Pre-existing depression was a consistent independent risk factor for intensive care unitrelated PTSD at both 3 and 12 months ${ }^{56}$. A lack of awareness of PTSD complaints is common and post-traumatic suffering is often silent, because other comorbid disorders and complaints are more prominent early in the clinical picture following traumatic experiences. Thus a depressive episode characterized as help-seeking signal or drug-pursuing behavior is frequently encountered ${ }^{57}$. Our findings could also partially be explained by a lack of awareness among patients and clinicians. Moreover, the knowledge, skills and ability to diagnose depressive disorders are more sophisticated than for PTSD in general physicians and public health workers. More people with depressive disorders are therefore likely to be detected and managed than those with PTSD ${ }^{58}$. Identifying those at the greatest risk of developing PTSD can allow adequate allocation of therapeutic resources. Finally, we also found the correlation between BPD and PTSD. The childhood traumatic experiences have been shown to associate with $\mathrm{BPD}^{59}$. It might explain why the patient with $\mathrm{BPD}$ was vulnerable to the subsequent development of PTSD in this study.

Limitations. It is worth considering a few limitations in the current study. First, some further factors related to the development of PTSD might not have been considered in our study, such as previous experience of trauma and personality traits. Furthermore, the later PTSD diagnosis could not be differentiated as the same or new course with the prior one. Therefore, previous history of PTSD was not included as a risk factor for incident case.

Second, the data used in the current study are limited to subjects attending the community-based integrated screening program. Whether PTSD cases identified in the screened cohort are generalizable to those in a non-screened cohort is unclear. 


\begin{tabular}{|c|c|c|c|c|c|c|c|c|c|}
\hline Variables & & $\mathrm{HR}^{*}$ & $95 \%$ & CI & p-value & aHR & $95 \%$ & CI & p-value \\
\hline \multirow{2}{*}{ Gender } & Male & 0.57 & 0.41 & 0.79 & 0.0009 & 0.70 & 0.47 & 1.05 & 0.0805 \\
\hline & Female & 1.00 & & & & 1.00 & & & \\
\hline Age (Mean (SD) & year & 0.97 & 0.96 & 0.98 & $<0.0001$ & 0.97 & 0.95 & 0.98 & $<0.0001$ \\
\hline \multirow{3}{*}{ Education } & Low & 0.48 & 0.29 & 0.79 & 0.0152 & 0.54 & 0.32 & 0.90 & 0.0641 \\
\hline & Medium & 0.75 & 0.53 & 1.06 & & 0.78 & 0.54 & 1.12 & \\
\hline & High & 1.00 & & & 1.00 & & & & \\
\hline \multirow{3}{*}{ Marriage } & Single & 1.31 & 0.84 & 2.04 & 0.4690 & & & & \\
\hline & Married & 1.00 & & & & & & & \\
\hline & Widow & 1.07 & 0.69 & 1.66 & & & & & \\
\hline \multirow{2}{*}{ Smoking } & No & 1.00 & & & 0.1042 & 1.00 & & & 0.0513 \\
\hline & Yes & 0.69 & 0.45 & 1.08 & & 0.64 & 0.40 & 1.00 & \\
\hline \multirow{2}{*}{ Drinking } & No & 1.00 & & & 0.0500 & & & & \\
\hline & Yes & 0.63 & 0.39 & 1.00 & & & & & \\
\hline \multirow{2}{*}{ Cancer } & No & 1.00 & & & 0.0140 & 1.00 & & & 0.0675 \\
\hline & Yes & 1.81 & 1.13 & 2.90 & & 1.57 & 0.97 & 2.52 & \\
\hline \multirow{2}{*}{ Diabetes } & No & 1.00 & & & 0.0239 & & & & \\
\hline & Yes & 1.52 & 1.06 & 2.18 & & & & & \\
\hline \multirow{2}{*}{ Hypertension } & No & 1.00 & & & 0.0046 & & & & \\
\hline & Yes & 1.68 & 1.18 & 2.42 & & & & & \\
\hline \multirow{2}{*}{ CVA } & No & 1.00 & & & 0.0028 & & & & \\
\hline & Yes & 2.06 & 1.28 & 3.30 & & & & & \\
\hline \multirow{2}{*}{ CVD } & No & 1.00 & & & $<0.0001$ & 1.00 & & & 0.0345 \\
\hline & Yes & 2.14 & 1.56 & 2.96 & & 1.45 & 1.03 & 2.04 & \\
\hline \multirow{2}{*}{ MetS } & No & 1.00 & & & 0.5024 & & & & \\
\hline & Yes & 0.84 & 0.51 & 1.39 & & & & & \\
\hline \multirow{2}{*}{ MDD } & No & 1.00 & & & $<0.0001$ & 1.00 & & & $<0.0001$ \\
\hline & Yes & 8.57 & 6.34 & 11.60 & & 7.03 & 5.02 & 9.85 & \\
\hline \multirow{2}{*}{ ANX } & No & 1.00 & & & 0.0004 & & & & \\
\hline & Yes & 3.04 & 1.65 & 5.60 & & & & & \\
\hline \multirow{2}{*}{ SUD } & No & 1.00 & & & 0.0011 & & & & \\
\hline & Yes & 3.91 & 1.73 & 8.84 & & & & & \\
\hline \multirow{2}{*}{ BPD } & No & 1.00 & & & $<0.0001$ & 1.00 & & & 0.0291 \\
\hline & Yes & 10.08 & 7.55 & 13.46 & & 1.86 & 1.07 & 3.24 & \\
\hline
\end{tabular}

Table 3. Estimated hazard ratios of demographic and clinical characteristics for incident PTSD by proportional hazards regression model. ${ }^{\star} 1$ Hazard ratios of education, marriage, smoking, drinking, physical illnesses, and mental disorders were adjusted by age and gender. 2 Education: Elementary schools or below (low); High school (medium); University (high). 3 HR, hazard ratio; aHR, adjusted hazard ratio. 4 CVA: cerebrovascular accidents; CVD: cardiovascular diseases; MetS: metabolic syndrome; SUD: substances use disorders; MDD: major depressive disorders; ANX: anxiety disorders; BPD: bipolar disorders.

Third, PTSD cases had to have been treated or diagnosed in a clinical setting in order to be in the NHID. This study likely underestimated the true number of PTSD cases, as many people might not seek care and go undiagnosed.

Clinical Implications. Our findings are consistent with the concept that subjects with CVD or affective disorders (both unipolar and bipolar disorders) may represent a high risk group for developing PTSD in the community. Traumatized people are far more likely to visit primary care physicians than mental health professionals to seek help for physical discomfort and mental symptoms. Therefore, general practitioners will play an important role in identifying and treating $\mathrm{PTSD}^{41,60}$.

\section{References}

1. Breslau, N. et al. Trauma and posttraumatic stress disorder in the community: the 1996 Detroit Area Survey of Trauma. Arch Gen Psychiatry. 55, 626-632 (1998).

2. U.S. Department of Veterans Affairs. How common is PTSD? (2015). Retrieved 21 March 2016 from Available at: http://www.ptsd. va.gov/public/PTSD-overview/basics/how-common-is-ptsd.asp (Accessed: 21th March 2016).

3. Kessler, R. C. et al. Lifetime prevalence and age-of-onset distributions of DSM-IV disorders in the National Comorbidity Survey Replication. Arch Gen Psychiatry. 62, 593-602 (2005).

4. Kessler, R. C., Chiu, W. T., Demler, O., Merikangas, K. R. \& Walters, E. E. Prevalence, severity, and comorbidity of 12-month DSMIV disorders in the National Comorbidity Survey Replication. Arch Gen Psychiatry. 62, 617-627 (2005).

5. Bromet, E., Sonnega, A. \& Kessler, R. C. Risk factors for DSM-III-R posttraumatic stress disorder: findings from the National Comorbidity Survey. Am J Epidemiol. 147, 353-361 (1998). 
6. Pietrzak, R. H., Goldstein, R. B., Southwick, S. M. \& Grant, B. F. Medical comorbidity of full and partial posttraumatic stress disorder in US adults: results from Wave 2 of the National Epidemiologic Survey on Alcohol and Related Conditions. Psychosom Med. 73, 697-707 (2011).

7. Kessler, R. C., Sonnega, A., Bromet, E., Hughes, M. \& Nelson, C. B. Posttraumatic stress disorder in the National Comorbidity Survey. Arch Gen Psychiatry. 52, 1048-60 (1995).

8. Wilder Schaaf, K. P. et al. Anxiety, depression, and PTSD following cardiac arrest: a systematic review of the literature. Resuscitation. 84, 873-877 (2013).

9. Mehnert, A., Nanninga, I., Fauth, M. \& Schäfer, I. Course and predictors of posttraumatic stress among male train drivers after the experience of 'person under the train' incidents. J Psychosom Res. 73, 191-196 (2012).

10. Nishi, D. et al. Incidence and prediction of post-traumatic stress disorder at 6 months after motor vehicle accidents in Japan. Psychosomatics. 54, 263-271 (2013).

11. Hamanaka, S. et al. Acute stress disorder and posttraumatic stress disorder symptoms among patients severely injured in motor vehicle accidents in Japan. Gen Hosp Psychiatry. 28, 234-241 (2006).

12. Tol, W. A., Barbui, C. \& van Ommeren, M. Management of acute stress, PTSD, and bereavement: WHO recommendations. JAMA. 310, 477-478 (2013).

13. Bromet, E. J. \& Gluzman, S. F. The state of mental health and alcoholism in Ukraine. In The WHO World Mental Health Surveys (ed. T. B. U. Ronald \& C. Kessler) pp. 431-445 Geneva Switzerland: Cambridge (2008).

14. Graaf Ron, de. \& Ormel, J. Mental disorders and service use in the Netherlands: Results from the European Study of the Epidemiology of Mental Disorders (ESEMeD). In The WHO World Mental Health Surveys (ed. T. B. U. Ronald \& C. Kessler) pp. 388-405 Geneva, Switzerland: Cambridge (2008).

15. Huang, Y., Liu, Z. \& Zhang, M. Mental disorders and service use in China. In The WHO World Mental Health Surveys (ed. T. B. U. Ronald \& C. Kessler) pp. 447-473 Geneva, Switzerland: Cambridge (2008).

16. Kawakami, N. \& Takeshima, T. Twelve-month prevalence, severity, and treatment of common mental disorders in communities in Japan. In The WHO World Mental Health Surveys (ed. T. B. U. Ronald \& C. Kessler) pp. 474-485 Geneva Switzerland: Cambridge (2008).

17. Bryant, R. A., Creamer, M., O'Donnell, M., Silove, D. \& McFarlane, A. C. A multisite study of initial respiration rate and heart rate as predictors of posttraumatic stress disorder. J Clin Psychiatry. 69, 1694-1701 (2008).

18. Mayou, R., Bryant, B. \& Ehlers, A. Prediction of psychological outcomes one year after a motor vehicle accident. Am J Psychiatry. $158,1231-1238(2001)$

19. O’Donnell, M. L., Creamer, M., Pattison, P. \& Atkin, C. Psychiatric morbidity following injury. Am J Psychiatry. 161, 507-514 (2004).

20. Schnyder, U., Moergeli, H., Klaghofer, R. \& Buddeberg, C. Incidence and prediction of posttraumatic stress disorder symptoms in severely injured accident victims. Am J Psychiatry. 158, 594-599 (2001).

21. Schnyder, U., Wittmann, L., Friedrich-Perez, J., Hepp, U. \& Moergeli, H. Posttraumatic stress disorder following accidental injury: rule or exception in Switzerland? Psychother Psychosom. 77, 111-118 (2008).

22. Shalev, A. Y. et al. Prospective study of posttraumatic stress disorder and depression following trauma. Am J Psychiatry. 155, 630-637 (1998).

23. Zatzick, D. F. et al. A nationwide US study of post-traumatic stress after hospitalization for physical injury. Psychol Med. 37, 1469-1480 (2007).

24. Brewin, C. R., Andrews, B. \& Valentine, J. D. Meta-analysis of risk factors for posttraumatic stress disorder in trauma-exposed adults. J Consult Clin Psychol. 68, 748-766 (2000).

25. Ozer, E. J., Best, S. R., Lipsey, T. L. \& Weiss, D. S. Predictors of posttraumatic stress disorder and symptoms in adults: a meta-analysis. Psychol Bull. 129, 52-73 (2003).

26. Koopman, C., Classen, C. \& Spiegel, D. Predictors of posttraumatic stress symptoms among survivors of the Oakland/Berkeley, Calif., firestorm. Am J Psychiatry. 151, 888-894 (1994).

27. Widom, C. S. Posttraumatic stress disorder in abused and neglected children grown up. Am J Psychiatry. 156, 1223-1229 (1999).

28. Creamer, M., Burgess, P. \& McFarlane, A. C. Post-traumatic stress disorder: findings from the Australian National Survey of Mental Health and Well-being. Psychol Med. 31, 1237-1247 (2001).

29. Breslau, N. Epidemiologic studies of trauma, posttraumatic stress disorder, and other psychiatric disorders. Can J Psychiatry. 47, 923-929 (2002).

30. Kibler, J. L., Joshi, K. \& Ma, M. Hypertension in relation to posttraumatic stress disorder and depression in the US National Comorbidity Survey. Behav Med. 34, 125-132 (2009).

31. Vieweg, W. V. et al. Posttraumatic stress disorder in male military veterans with comorbid overweight and obesity: psychotropic, antihypertensive, and metabolic medications. Prim Care Companion J Clin Psychiatry. 8, 25-31 (2006).

32. Chang, J. C. et al. A population-based cohort study to elucidate temporal relationship between schizophrenia and metabolic syndrome (KCIS no. PSY3). Schizophr Res. 151, 158-164 (2013).

33. Chang, J. C. et al. Metabolic syndrome and the risk of suicide: a community-based integrated screening samples cohort study. Psychosom Med. 75, 807-814 (2013).

34. Chen, T. H. et al. Community-based multiple screening model: design, implementation, and analysis of 42,387 participants. Cancer. 100, 1734-1743 (2004).

35. Wang, P. E., Wang, T. T., Chiu, Y. H., Yen, A. M. \& Chen, T. H. Evolution of multiple disease screening in Keelung: a model for community involvement in health interventions? J Med Screen. 13 (Suppl. 1), S54-S58 (2006).

36. Expert Panel on Detection Evaluation and Treatment of High Blood Cholesterol In Adults. Executive Summary of The Third Report of The National Cholesterol Education Program (NCEP) Expert Panel on Detection, Evaluation, And Treatment of High Blood Cholesterol In Adults (Adult Treatment Panel III). JAMA. 285, 2486-2497 (2001).

37. World Health Organization. The Asia-Pacific perspective: redefining obesity and its treatment. WHO: Geneva (2000).

38. Roberts, A. L., Gilman, S. E., Breslau, J., Breslau, N. \& Koenen, K. C. Race/ethnic differences in exposure to traumatic events, development of post-traumatic stress disorder, and treatment-seeking for post-traumatic stress disorder in the United States. Psychol Med. 41, 71-83 (2011).

39. Liao, S. C. et al. Low prevalence of major depressive disorder in Taiwanese adults: possible explanations and implications. Psychol Med. 42, 1227-1237 (2012)

40. Chen, S. L. S. et al. A 10-year follow-up study on suicidal mortality after 1999 Taiwan Earthquake. J Psychiatr Res. 79, 42-49 (2016).

41. Greene, T., Neria, Y. \& Gross, R. Prevalence, detection and correlates of PTSD in the primary care setting: A systematic review. J Clin Psychol Med Settings. 23, 160-80 (2016).

42. Bromet, E. J. et al. Post-traumatic stress disorder associated with natural and human-made disasters in the World Mental Health Surveys. Psychol Med. 30, 1-15 (2016)

43. Spitzer, C. et al. Trauma, posttraumatic stress disorder, and physical illness: findings from the general population. Psychosom Med. 71, 1012-1017 (2009).

44. Edmondson, D. \& Cohen, B. E. Posttraumatic stress disorder and cardiovascular disease. Prog Cardiovasc Dis. 55, 548-556 (2013).

45. Coughlin, S. S. Post-traumatic stress disorder and cardiovascular disease. Open Cardiovasc Med J. 5, 164-170 (2011).

46. Boscarino, J. A. Post-traumatic stress disorder and cardiovascular disease link: time to identify specific pathways and interventions. Am J Cardiol. 108, 1052-1053 (2011). 
47. Wentworth, B. A. et al. Post-traumatic stress disorder: a fast track to premature cardiovascular disease? Cardiol Rev. 21, 16-22 (2013).

48. Bedi, U. S. \& Arora, R. Cardiovascular manifestations of posttraumatic stress disorder. J Natl Med Assoc. 99, 642-649 (2007).

49. Dedert, E. A., Calhoun, P. S., Watkins, L. L., Sherwood, A. \& Beckham, J. C. Posttraumatic stress disorder, cardiovascular and metabolic disease: a review of the evidence. Ann Behav Med. 39, 61-78 (2010).

50. Jordan, H. T., Miller-Archie, S. A., Cone, J. E., Morabia, A. \& Stellman, S. D. Heart disease among adults exposed to the September 11, 2001 World Trade Center disaster: results from the World Trade Center registry. Prev Med. 53, 370-376 (2011).

51. von Känel, R. et al. Evidence for low-grade systemic proinflammatory activity in patients with posttraumatic stress disorder. $J$ Psychiatr Res. 41, 744-752 (2007).

52. Shemesh, E. et al. Posttraumatic stress, nonadherence, and adverse outcome in survivors of a myocardial infarction. Psychosom Med. 66, 521-526 (2004)

53. Posta, L. M., Zoellnerb, L. A., Youngstromc, E. \& Feenya, N. C. Understanding the relationship between co-occurring PTSD and MDD: Symptom severity and affect. J Anxiety Disord. 25, 1123-1130 (2011).

54. Warren, A. M. et al. Posttraumatic stress disorder following traumatic injury at 6 months: associations with alcohol use and depression. J Trauma Acute Care Surg. 76, 517-522 (2014).

55. Tiihonen Möller, A., Bäckström, T., Söndergaard, H. P. \& Helström, L. Identifying risk factors for PTSD in women seeking medical help after rape. PLoS ONE. 9, e111136 (2014).

56. Patel, M. B. et al. Incidence and Risk Factors for ICU-related Posttraumatic Stress Disorder In Veterans and Civilians. Am J Respir Crit Care Med. 193, 1373-81 (2016)

57. Auxéméry, Y. Posttraumatic stress disorder (PTSD) as a consequence of the interaction between an individual genetic susceptibility, a traumatogenic event and a social context. Encephale. 38, 373-380 (2012).

58. Bolduc, A. et al. Identification and referral of patients at risk for Post-traumatic Stress Disorder: A literature review and retrospective analysis. Am Surg. 81, 904-908 (2015).

59. Agnew-Blais, J. \& Danese, A. Childhood maltreatment and unfavourable clinical outcomes in bipolar disorder: a systematic review and meta-analysis. Lancet Psychiatry. 3, 342-9 (2016).

60. Yehuda, R. Post-traumatic stress disorder. N Engl J Med. 346, 108-114 (2002).

\section{Author Contributions}

Dr. Jung-Chen Chang contributed to the first draft of the manuscript and analyzing the data and statistical analysis as well as providing suggestions to the final draft of the manuscript. Dr. Amy Ming-Fang Yen assisted in managing and analyzing the data and statistical analysis and providing critical comments on methodological aspects of the article. Dr. Sam Li-Sheng Chen, Dr. Sherry Yueh-Hsia Chiu and Dr. Jean Ching-Yuan Fann assisted the data collection and management as well as provided critical feedback to the study design and analysis. Professor Tony Hsiu-Hsi Chen played a major leading role in designing the study, and revising the manuscript. All authors contributed to and have approved the final manuscript. Dr. Chau-Shoun Lee contributed to the reference synthesis, the clinical interpretation of findings, and major revisions of the manuscript.

\section{Additional Information}

Competing financial interests: The authors declare no competing financial interests.

How to cite this article: Chang, J.-C. et al. Comorbid diseases as risk factors for incident posttraumatic stress disorder (PTSD) in a large community cohort (KCIS no.PSY4). Sci. Rep. 7, 41276; doi: 10.1038/srep41276 (2017).

Publisher's note: Springer Nature remains neutral with regard to jurisdictional claims in published maps and institutional affiliations.

(c) (i) This work is licensed under a Creative Commons Attribution 4.0 International License. The images or other third party material in this article are included in the article's Creative Commons license, unless indicated otherwise in the credit line; if the material is not included under the Creative Commons license, users will need to obtain permission from the license holder to reproduce the material. To view a copy of this license, visit http://creativecommons.org/licenses/by/4.0/

(C) The Author(s) 2017 